\title{
The MAGNUM survey: a high-resolution study of the complex nuclear environment of local Seyfert galaxies
}

\author{
Mingozzi M.*1,2, Venturi, G. ${ }^{\dagger 3,2}$, Mannucci F. ${ }^{\ddagger 2}$, Marconi A. ${ }^{\S 4,2}$, and Cresci G. ${ }^{\llbracket 2}$ \\ ${ }^{1}$ INAF - Osservatorio Astronomica di Padova, Padova, Italy \\ ${ }^{2}$ INAF - Osservatorio Astrofisico di Arcetri, Florence, Italy \\ ${ }^{3}$ PUC - Pontificia Universitad de Chile, Santiago de Chile \\ ${ }^{4}$ Università di Firenze, Dipartimento di Fisica e Astronomia, Firenze, Italy
}

\begin{abstract}
The central regions of Seyfert galaxies, comprising broad and narrow line regions and the inner parts of galaxy disk and bulge, is characterized by a complex interplay among many physical effects. Specifically, it is shaped by the influence of the central black hole, producing ionization by an hard continuum and gas outflows. The integral-field spectrograph MUSE at the ESO VLT allows to carry out a detailed study of these regions to obtain their ionization, dynamical, and metallicity properties. Here we present some highlights of the MAGNUM survey which is designed to study the central regions of a sample of nearby $(\mathrm{D}<50 \mathrm{Mpc})$ Seyfert galaxies. We describe the rationale of the survey, the data analysis techniques used to extract information on ionization and dynamics, and the results for one galaxy, Centaurus A.
\end{abstract}

\section{Introduction}

A variety of physical processes shape the observed distribution of galaxy properties: some of them account for galaxy growth (e.g., star formation, SF; galaxy merging), while others regulate this growth (e.g., energetic feedback from supernovae, SN and active galactic nuclei, AGN; ultraviolet, $\mathrm{UV}$, radiation background). Indeed the evolution of a galaxy is a matter of synergy among these diverse physical processes. Metal poor gas is accreted from the intergalactic medium (IGM), the dilute reservoir that permeates the space between galaxies (McQuinn, 2016), triggering SF inside the galaxy. However, gas accretion and SF can also be the consequence of major and/or minor mergers. Stellar evolution, via SN explosions and stellar winds, provides chemical enrichment of the gaseous component of galaxies, referred to as the interstellar medium (ISM) or the diffuse matter (Klessen \& Glover, 2016). Part of this enriched gas leaves the galaxy through galactic winds, enriching the circumgalactic medium (CGM), a large gas halo extending by hundreds of $\mathrm{kpc}$ in which galaxies are embedded, or the IGM, while the rest is recycled several times, in several stellar generations. Also dust plays a role in the physics of ISM, since it locks and releases metals, being continuously created and destroyed. Finally, the presence of a central AGN can largely influence the properties of the ISM and of the CGM, by heating, compressing and/or removing the gas through AGN-driven winds and jets (feedback). These processes that cause this gas flow in and out of galaxies providing the fuel both for SF and black hole (BH) accretion are known as the galaxy baryon cycle. Overall, since the ISM is the primary "repository" of galaxies, the study of its properties can give information on all the processes listed above (Maiolino \& Mannucci, 2019).

Here we present the early results of the MAGNUM (Measuring AGN under MUSE microscope) survey (P.I. A. Marconi), using the IFU spectrograph MUSE on ESO/VLT to study the inner regions

*matilde.mingozzi@inaf.it

${ }^{\dagger}$ gventuri@astro.puc.cl

${ }^{\ddagger}$ filippo.mannucci@inaf.it, Corresponding author

§alessandro.marconi@unifi.it

๑giovanni.cresci@inaf.it 
of a number of local AGNs with the aim of disentangling all these effects. The high spatial resolution obtained by MUSE ( $\sim 0.6-0.8$ arcsec $)$ and the large spatial coverage $(\sim 1$ arcmin $)$ will allows us to derive a complete picture of these galaxies, in terms of metallicity, ionization properties, dynamical status, and stellar populations.

This contribution is based on a number of published papers, namely Cresci et al. (2015b), Venturi et al. (2017), Venturi et al. (2018), and Mingozzi et al. (2019), and on unpublished material from M. Mingozzi's Phd Thesis (2019, University of Bologna), and G. Venturi's PhD Thesis (2018, University of Florence).

\section{Galaxy sample, observations, and data analysis}

MAGNUM galaxies have been selected to be observable from Paranal Observatory and with a luminosity distance $\mathrm{D}<50 \mathrm{Mpc}$. In Venturi et al. (2020, in prep.) we present our sample, explaining in detail the selection criteria, data reduction and analysis, and investigating the kinematics of the ionized gas. We observed nine Seyfert galaxies, namely Centaurus A, Circinus, NGC 4945, NGC 1068, NGC 1365, NGC 1386, NGC 2992, NGC 4945 and NGC 5643. Each galaxy was observed for 0.5-1 hours.

The final datacube on each galaxy consists of $\sim 300 \times 300$ spaxels, for a total of over 90000 spectra with a spatial sampling of $0.2 " \times 0.2$ " and a spectral resolution going from 1750 at $4650 \AA$ to 3750 at $9300 \AA$. The FOV of $1^{\prime} \times 1^{\prime}$ covers the central part of the galaxies, spanning from 1 to $10 \mathrm{kpc}$, according to their distance. The average seeing of the observations, derived directly from foreground stars in the final datacubes, is $\sim 0.6-0.8 "$. The datacubes were analysed making use of a set of custom python scripts in order to fit and subtract the stellar continuum in each single-spaxel spectrum and fit the emission lines with multiple Gaussian components where needed. Here we summarise the main steps of the data analysis.

\section{Stellar continuum fitting}

First of all, a Voronoi adaptive binning, that is a technique that allows to obtain bins with different spatial extension but having a minimum signal-to-noise ratio $(\mathrm{S} / \mathrm{N})$ ratio in the data, was performed in order to achieve an average $\mathrm{S} / \mathrm{N}$ per wavelength channel of 50 on the continuum under $5530 \AA$, where stellar features are more prominent. This threshold allows us to fit also the fainter regions of the FOV, preserving the maximum spatial resolution. In order to fit the stellar continuum and derive the systemic velocity and velocity dispersion of the stellar absorption lines, we applied the Penalized Pixel-Fitting (PPXF; Cappellari \& Emsellem 2004) code on the Voronoi-binned spaxels. Basically, this fast and simple algorithm recovers galaxy stellar kinematics by fitting a template to an observed spectrum, applying the maximum penalized likelihood formalism. We employed as a template a linear combination of Vazdekis et al. (2010) synthetic spectral energy distributions for single stellar population models in the wavelength range $4750-7500 \AA$. Note that at this stage our aim is only to reproduce the shape of the stellar features and derive the bulk velocity and velocity dispersion of the stellar population, and not to get more detailed physical information from the models. Instead, a linear baseline was used to fit the local continuum around the [S III] $\lambda 9069$ emission line, since this line is not contaminated by underlying absorptions. The main emission lines lying in the selected wavelength range $(\mathrm{H} \beta-[\mathrm{O}$ III $] \lambda \lambda 4959,5007$ - [O I $] \lambda \lambda 6300,64-\mathrm{H} \alpha-[\mathrm{N}$ II $] \lambda \lambda 6548,84-[\mathrm{S}$ II $] \lambda \lambda 6717,31)$ were included in the fit as well, in order to better constrain the absorption underlying the Balmer lines. Fainter lines and regions affected by sky residuals were masked. The emission lines were fitted with one, two, three, or four Gaussian components according to the shape of the line profile, tying the velocity and width of each component to be the same for all the lines and leaving the intensities free to vary, apart from the [O III], [O I] , and [N $\mathrm{NI}$ ] doublets, where an intrinsic ratio of 0.333 between the two lines was used (see e.g. Storey \& Zeippen 2000).

A reduced $\chi^{2}\left(\tilde{\chi}^{2}\right)$ selection to the different fits is made in each spaxel, in order to define where multiple components were really needed to reproduce the observed spectral profiles, with the idea of keeping the number of parameters of the fit as low as possible and using multiple components only in 
case of complex non-Gaussian line profiles. This happens mainly in the central parts of the galaxies and in the outflowing cones.

The stellar continuum fit included also a multiplicative and an additive polynomials of degree $m$ and $a$ (with $7<m<10$ and $a=2$ ) to account for any spectrum deformation not present in the models (e.g. reddening). Finally, the fitted stellar continuum in each Voronoi bin was subtracted spaxel by spaxel, rescaling the modelled continuum emission in each bin to the median of the observed continuum of each spaxel.

NGC 1365 and NGC 2992 host a Seyfert 1 nucleus. For these galaxies we subtracted the contribution from the BLR assumed to be spatially unresolved and spread across the FoV due to the seeing.

\section{Emission line fitting}

From the continuum-subtracted cube, we generated a spatially smoothed data cube and a Voronoibinned data cube. The former is obtained by spatially smoothing the cube with a Gaussian kernel having $\sigma=1$ spaxel (i.e. 0.2"), without degrading significantly the spatial resolution, given the observational seeing. The latter, produced by applying a Voronoi binning in the range $\sim 4850-4870 \AA$ in the vicinity of $\mathrm{H} \beta$, requiring an average $\mathrm{S} / \mathrm{N}$ per wavelength channel of at least 3 , can be used to make quantitative comparisons among the galaxies.

Finally, in each spaxel (or bin in the Voronoi-binned cube), the gas emission lines (H $\beta$ - $[\mathrm{O}$ III $] \lambda \lambda 4959$, 5007 - [O I $] \lambda \lambda 6300,64-\mathrm{H} \alpha-[\mathrm{N} \mathrm{II}] \lambda \lambda 6548,84-[\mathrm{S} \mathrm{II}] \lambda \lambda 6717,31)$ were fitted in the same spectral range used for the stellar fitting, while the residual continuum was fitted with a linear baseline, free to vary to account for small deviations of the overall residuals from null value. To do this, we employed the MPFIT procedure (Markwardt, 2009), taking into account one, two, three, or four Gaussians to reproduce the emission line profile, tying the velocity and widths of each component to be the same for all the lines and leaving the intensities free to vary (apart from the [O III], [O I], and [N II] doublets), as described above. Note that the width of all the emission lines was forced to be greater than MUSE spectral resolution $(\sim 65 \mathrm{~km} / \mathrm{s}$ at $[\mathrm{O}$ III]-H $\beta$ wavelengths; $\sim 50 \mathrm{~km} / \mathrm{s}$ at $[\mathrm{N} \mathrm{II}]-\mathrm{H} \alpha-[\mathrm{S} \mathrm{II}]$ wavelengths $)$. Then, the $[\mathrm{S}$ III] $] \lambda 9069$ emission line fit is performed analogously, fixing the velocity and width of each of its Gaussian components to the values obtained for the other emission lines in the previous fit. The same $\tilde{\chi}^{2}$ selection described above is applied to choose the best-fit in each spaxel (bin). Finally, the flux of each emission line is the sum of the integrated fluxes of the adopted Gaussians.

\section{Kinematic maps}

The analysis of MUSE data described above leads to trace the kinematics of the stars and the warm ionized gas. As an example, Fig. 1 shows the kinematic maps of one galaxy of the sample, NGC 1365, that we investigated in detail in Venturi et al. (2018), to visually appreciate the outflow shape and location within the galaxy.

The stellar velocity map (Fig. 1a) has been obtained from the fit of stellar continuum carried out on the Voronoi-binned data cube, and clearly shows the rotation of the stars in the galactic disc, approaching the observer to the NE and receding to the SW direction. The twisted shape in the central region corresponds to the boundary where the velocity changes sign and is likely due to the presence of the bar.

The $\left[\mathrm{O}\right.$ III] velocity map (Fig. $1 \mathrm{~b}$ ) instead is the $1^{\text {st }}$-order moment of the $[\mathrm{O} \mathrm{III}]$ total line profile resulting from the fit of the star-subtracted smoothed data cube. In addition to the rotational field, it shows other complex motions, that can be isolated and highlighted by subtracting spaxel-by-spaxel the stellar rotation (Fig. 1c). This reveals a clumpy double-conical outflow approximately in the SENW direction, with the SE and NW cone having approaching and receding velocities, respectively. The shape and structure of the outflow are well-traced by the [O III] W70 velocity width (Fig. 1d), that is the difference between the velocities including $85 \%$ and $15 \%$ of the flux of the total fitted line profile. High W70 values come from the regions of the outflow, composed by the kinematic components belonging to disc, bar and outflow. 

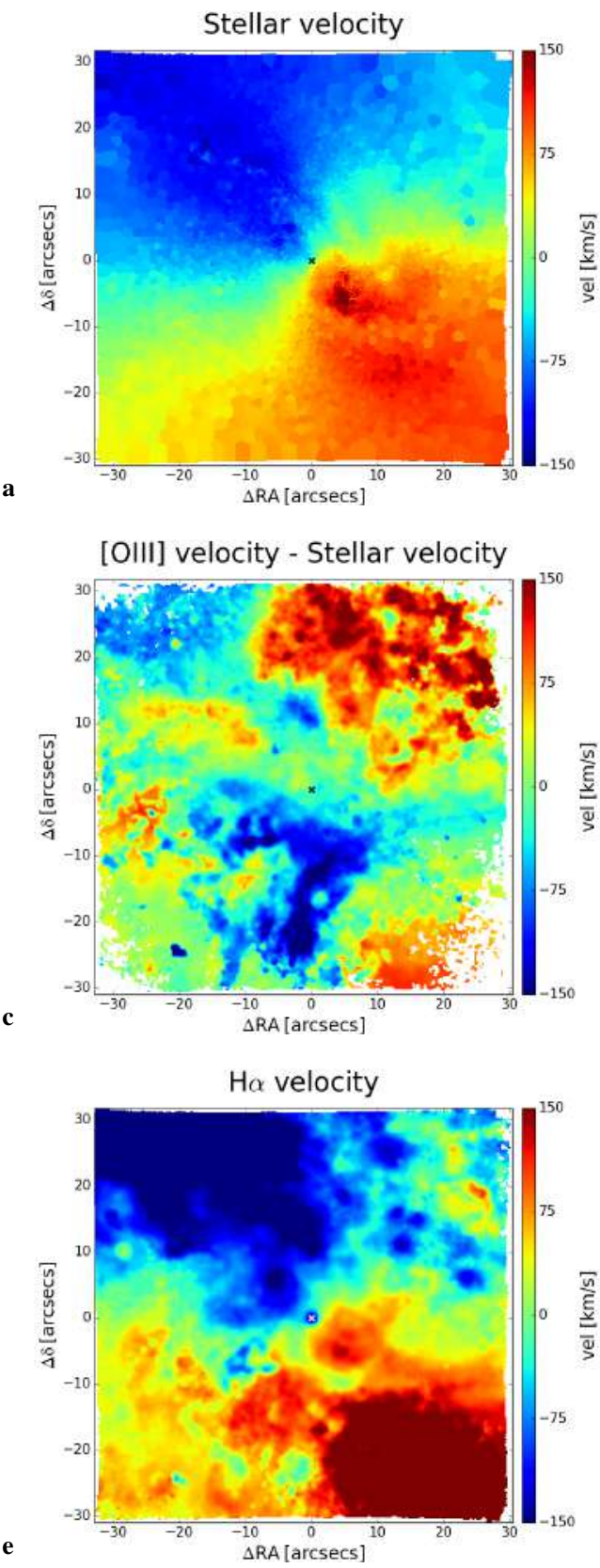
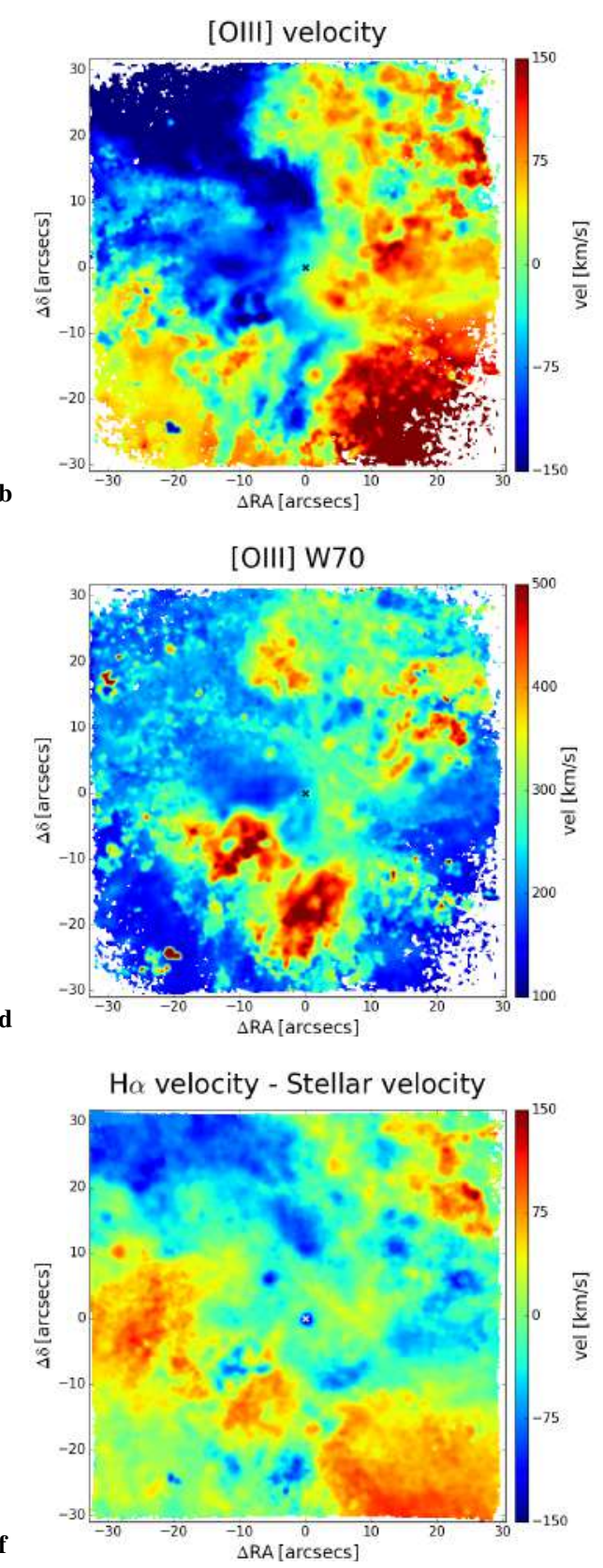

Figure 1. (a) Stellar velocity map of NGC 1365, with respect to the systemic velocity $\left(1630 \mathrm{~km} \mathrm{~s}^{-1}\right)$. (b) [O III] velocity map with respect to the systemic velocity. This map has been re-smoothed with a $\sigma=1$ spaxel Gaussian kernel for a better visual output, and a signal-to-noise cut of 3 was applied (this holds for the other maps reported in panels c, d, e, f). (c) [O III] velocity subtracted spixel-by-pixel by the stellar velocity. (d) [O III] W70 (i.e. difference between the 85th-percentile and 15th-percentile velocities of the fitted line profile) map. (e) $\mathrm{H} \alpha$ velocity map with respect to the systemic velocity. (f) $\mathrm{H} \alpha$ velocity map spaxel-by-spaxel subtracted by the stellar velocity. East is to the left. This figure is taken from Venturi et al. (2018).

The kinematics of the ionized gas in the disk can be better inspected from the $\mathrm{H} \alpha$ velocity map (see Venturi et al. 2018 for more details). Fig. 1e and Fig. 1f show the H $\alpha$ velocity map with respect to the rest frame velocity and to the stellar velocity, respectively. The $\mathrm{H} \alpha$ kinematics is primarily dominated by two thick lanes aligned with the bar in the NE-SW direction, having opposite velocities in excess to stellar rotation. This non-circular motions may indicate an inflow of material along the bar of NGC 1365. 


\section{Gas properties: disk versus outflow}

Excitation and ionization conditions, as well as dust attenuation of the ISM in each MAGNUM galaxy can be investigated through specific emission-line ratios. Thanks to integral field spectrographs (IFUs), we explore the different properties of the disk and the outflow of our galaxies by separating and analysing the different gas components across the MUSE FOV.

Specifically, we define velocity channels of $\sim 50 \mathrm{~km} / \mathrm{s}$, ranging from $-1000 \mathrm{~km} / \mathrm{s}$ to $+1000 \mathrm{~km} / \mathrm{s}$

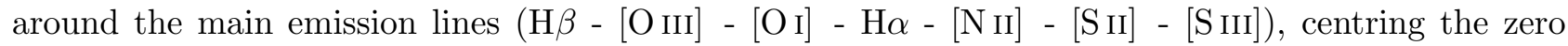
velocity to the measured stellar velocity in each spaxel of the MUSE FOV for all the galaxies, since the stellar velocity is generally a good approximation of the gas velocity in the disk. In order to derive the flux in each velocity bin, we integrate the fitted line profile within each velocity channel of the emission lines taken into account. However, Centaurus A is characterised by a strong misalignment between stars and gas (e.g. Morganti 2010), and thus we consider the global systemic velocity $\left(v_{\text {sys }}=547 \mathrm{~km} / \mathrm{s}\right)$ as a reference for the gas disk velocity.

We associate the velocity channels close to the core of the lines in the fitted line profile with the disk (hereafter disk component), and the sum of the redshifted and blueshifted channels with the outflow (hereafter outflow component). Specifically, the disk component comprises velocity in the range $-100 \mathrm{~km} / \mathrm{s}<v<100 \mathrm{~km} / \mathrm{s}$ for Circinus, NGC 1365, and NGC 1386, and $-150 \mathrm{~km} / \mathrm{s}<$ $v<150 \mathrm{~km} / \mathrm{s}$ for the other galaxies, while the outflow component is associated with velocities $v>$ $+150 \mathrm{~km} / \mathrm{s}$ and $v<-150 \mathrm{~km} / \mathrm{s}$ for Circinus, NGC 1365 and NGC 1386, $v>+250 \mathrm{~km} / \mathrm{s}$ and $v<-250 \mathrm{~km} / \mathrm{s}$ for NGC 1068, and $v>+200 \mathrm{~km} / \mathrm{s}$ and $v<-200 \mathrm{~km} / \mathrm{s}$ for the other galaxies. These values are chosen after an accurate spaxel-by-spaxel analysis of the spectra in the outflowing regions of the galaxies. Overall, the disk component represents the low-velocity component of the ionized gas, which is rotating similarly to the stars, while the outflow component (i.e. the high-velocity component) is moving faster than the stellar velocity, and is partly blueshifted and partly redshifted with respect to it. Since we are looking at the central regions of AGN galaxies, the narrow line region (NLR) is present in both the disk and in the outflow components.

Because the $\mathrm{H} \alpha$ emission is in general dominated by the disc, while the outflow is enhanced in [O III] (e.g. Venturi et al. 2018), in Fig. 2 we show the $\mathrm{H} \alpha$ disk component flux maps, superimposing the [O III] $\lambda 5007$ outflow component flux contours (not corrected for dust reddening) for all the galaxies, using the method described above to separate the two components. The blueshifted and redshifted outflowing components are indicated in blue and red, respectively. For each velocity bin, we only select the spaxels with a $\mathrm{S} / \mathrm{N}>5$, computed by dividing the integrated flux in the velocity channels by the corresponding noise. The noise is estimated from the standard deviation of the data-model residuals of the fit around each line (within a range about 60 to $110 \AA$ wide, depending on the line). Looking at Fig. 2, it can be seen that the disk flux maps and the outflow contours are clearly different from each other. The outflow component is often extended in a kpc-scale conical or biconical distribution, as can be clearly seen in Circinus (north-west cone), IC 5063 (north-west and south-east cones), NGC 2992 (north-west and south-east cones), NGC 4945 (north-west and south-east cones), and NGC 5643 (east and west cones). In Centaurus A the outflow component is mainly distributed in two cones (direction north-east and south-west) in the same direction of the extended double-sided jet revealed both in the radio and X-rays (e.g. Hardcastle et al. 2003), and located perpendicularly with respect to the gas in the disk component. Unfortunately, since for this galaxy the stellar velocity cannot be taken as a reference, in some regions we underestimate the gas velocity of the disk component. Consequently, a portion of the disk is still present in the outflowing component. Also in NGC 1365, the outflow component flux map has a biconical shape extended in the south-east and north-west directions, while the disk component appears to be completely different, being dominated by an elongated circumnuclear SF ring and by the bar.

Unfortunately, similar to Centaurus A, a portion of the disk is still present in our outflowing gas selection, because of the high velocity reached by the gas along the bar (see Venturi et al. 2018 for more details). NGC 1068 is almost face-on, allowing us to admire the spiral arms of the disc, traced by the disk component, and preventing the outflowing component from having a clear biconical distribution, but to be broadly extended in all the inner region of the observed FOV. Both the ionized and molecular outflow already observed in this galaxy are observed in the north-east and south-west directions (e.g. 

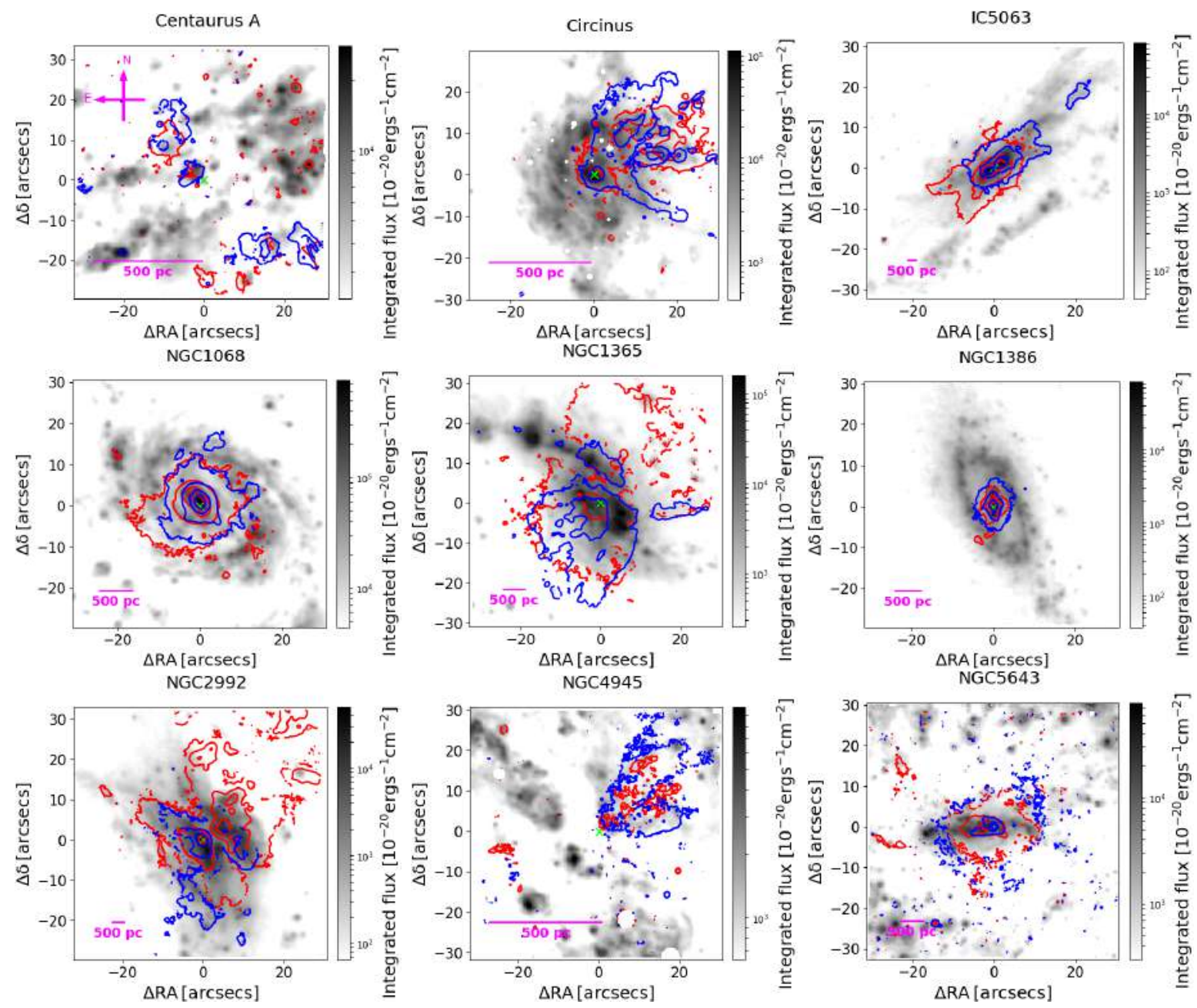

Figure 2. $\mathrm{H} \alpha$ disk component flux maps (not corrected for dust reddening) for all the galaxies, namely Centaurus A, Circinus, IC 5063, NGC 1068, NGC 1365, NGC 1386, NGC 2992, NGC 4945, and NGC 5643. [O III] $\lambda_{5007}$ outflow component flux contours are superimposed for all the galaxies. The blueshifted and redshifted outflow emission (in blue and red, respectively) is often extended in a kiloparsec-scale conical or biconical distribution. For each velocity bin, we show only the spaxels with a $\mathrm{S} / \mathrm{N}>5$. The magenta bar represents a physical scale of $\sim 500 \mathrm{pc}$. East is to the left, as shown in the first image on the left. The white circular regions are masked foreground stars. The cross marks the position either of the Type 1 nucleus (i.e. peak of the broad $\mathrm{H} \alpha$ emission), if present, or the peak of the continuum in the wavelength range $6800-7000 \AA$.

Cecil et al. 2002, García-Burillo et al. 2014). Finally, in NGC 1386 the outflow component does not show a well-defined conical distribution, but appears to be located in the very inner region of the galaxy, with two elongated structures to the north and south of the nucleus, corresponding to nuclear bipolar outflows already revealed by Ferruit et al. (2000) and Lena et al. (2015).

Note that in almost all galaxies the (bi)conical outflow is detected in both its blueshifted and redshifted components, which are often overlapping. For example, this can be clearly seen in NGC 4945, where the north-west cone has approaching velocities at its edges and receding ones around its axis, while the south-east cone has the opposite behaviour, with receding velocities at its edges and approaching ones around its axis. This was already revealed by Venturi et al. (2017) through the kinematic maps of the $[\mathrm{N}$ II] line emission.

\section{Spatially and kinematically resolved BPT}

Spatially and kinematically resolved Baldwin-Phillips-Terlevich (BPT) diagrams allow us to explore the dominant contribution to ionization in each spaxel in the disk and outflow components 
separately (a similar approach has been used by e.g. Westmoquette et al. 2012, McElroy et al. 2015, and Karouzos et al. 2016).

The left and middle panels of Fig. 3 show the [N II]- and [S II]-BPT diagrams of Circinus for the disk and outflow components, respectively. For each velocity bin, we select only the spaxels with a $\mathrm{S} / \mathrm{N}>5$, computed by dividing the integrated flux in the velocity channels for each emission line by the corresponding noise. The dashed curve is the boundary between star forming galaxies and AGN defined by Kauffmann et al. (2003) (ka03), while the solid curve is the theoretical upper limit on SF line ratios found by Kewley et al. (2001) (ke01). The dotted line, instead, is the boundary between Seyferts and LI(N)ERs introduced by Kewley et al. (2006) (ke06). The dominant source of ionization is colour-coded: shades of blue for SF, green for intermediate regions in the [N II]-BPT and $\mathrm{LI}(\mathrm{N}) \mathrm{ER}$ in the $[\mathrm{SII}]-\mathrm{BPT}$, and red for AGN-like ionizing spectra, as a function of the $\mathrm{x}$-axis line ratios (darker shades means higher x-axis line ratios). The LI(N)ER-like excitation can be due either to shock excitation (e.g. Dopita \& Sutherland 1995) or to hard-X radiation coming from AGN and to hot evolved (post-asymptotic giant branch) stars (e.g. Belfiore et al. 2016). The corresponding position on the map of the outflowing gas component, colour-coloured based on the different sources of ionization, is shown in the right panels of Fig. 3. In the background of all the pictures (black dots in the BPTs and shaded grey in the corresponding maps), we show the disk and outflow components together to allow us a better visual comparison.
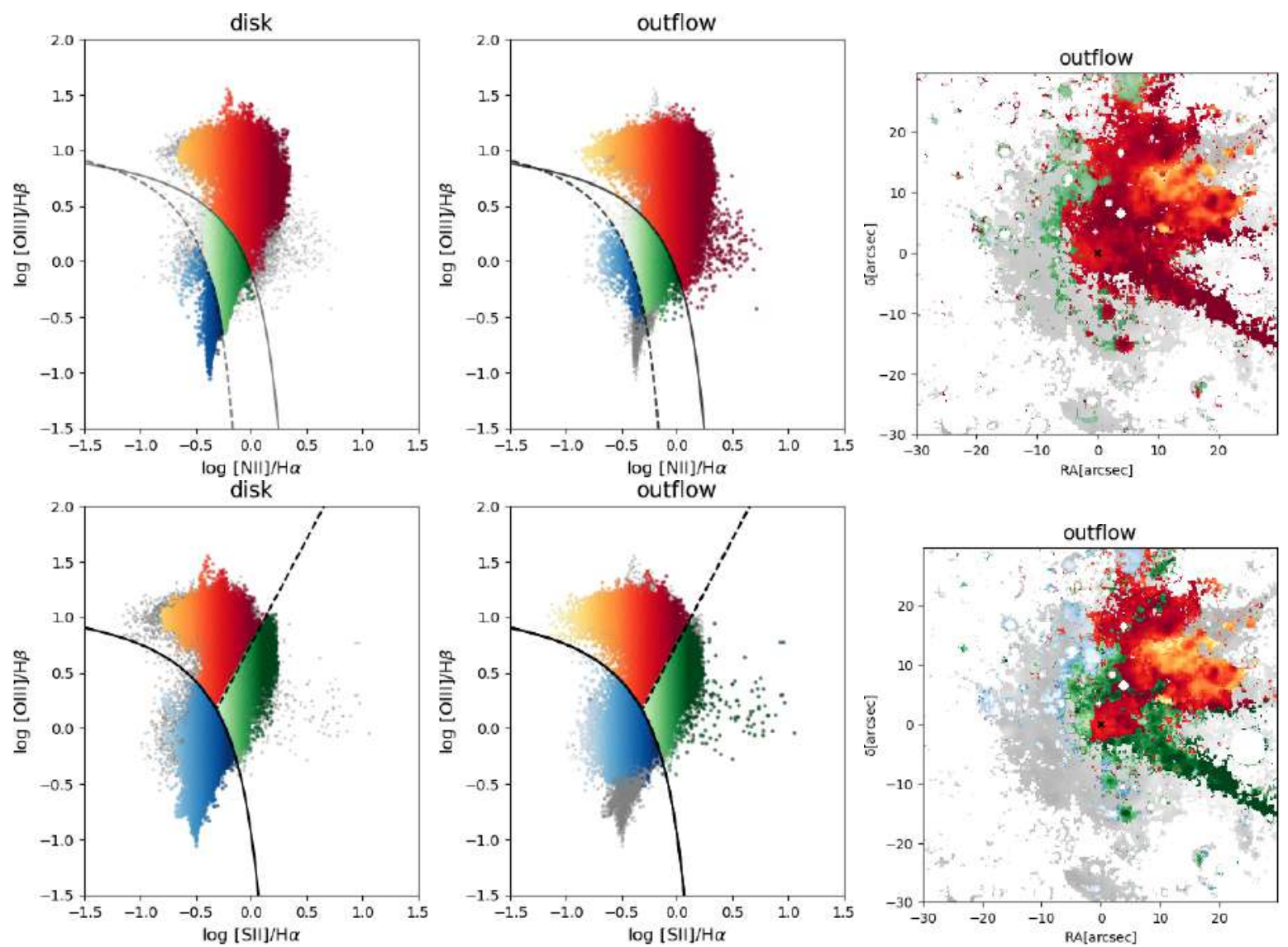

Figure 3. Left and central panels: [N II]- and [S II]-BPT diagrams for the disk and outflow components, on the left and on the right respectively, of Circinus. Blue denotes SF-dominated regions, green intermediate regions in the $[\mathrm{NII}]-\mathrm{BPT}$ and $\mathrm{LI}(\mathrm{N}) \mathrm{ER}$ in the $[\mathrm{SII}]-\mathrm{BPT}$, and red AGN-like ionized spectra, colours-coded as a function of the $\mathrm{x}$-axis line ratios (darker shades means higher $\mathrm{x}$-axis line ratios). The black dashed and solid curves, and the black dotted line show the ka03, ke01 and ke06 boundaries, respectively. Right panels: [N II]- and [S II]-BPT maps of the outflowing gas component, colour-coded according to BPTs. In the background of all the pictures (grey dots in the BPTs and shaded grey in the corresponding maps), we show the disk and outflow component together. For each velocity bin, we select only the spaxels with a $S / N>5$ for all the lines involved. 
Looking at Fig. 3, we note that the outflow spans a wider range of $[\mathrm{N} \mathrm{II}] / \mathrm{H} \alpha$ and $[\mathrm{S} \mathrm{II}] / \mathrm{H} \alpha$, including lower and higher values compared to the disk. Specifically, the highest and lowest values of lowionization line ratios (LILrs; i.e. $[\mathrm{NII}] / \mathrm{H} \alpha$ and $[\mathrm{SII}] / \mathrm{H} \alpha$ ), displayed in dark red and orange, are prominent in the AGN/LI(N)ER-dominated outflow component, while they are not observable in the disk component. As described in detail in Mingozzi et al. (2019), these features are visible in almost all the MAGNUM sample, in which, in general, the highest LILrs trace the inner parts along the axis of the emitting cones, where the $[\mathrm{S} \mathrm{III}] /[\mathrm{S}$ II $]$ line ratio is enhanced (i.e. high excitation), while the lowest LILrs follow the cone edges and/or the regions perpendicular to the axis of the outflow, characterised also by a higher $[\mathrm{O}$ III] velocity dispersion.

\section{Centaurus A: an ideal local laboratory to study AGN positive feedback}

This galaxy is the result of a recent merger between an early-type elliptical and a spiral galaxy (e.g. Israel 1998). Indeed, it is characterised by a stellar-dominated almost circular component and by a dusty and gaseous thick lane approximately in the SE-NW direction, containing the majority of molecular, ionized and neutral atomic gas (Morganti, 2010). Centaurus A galaxy shows a characteristic double-sided jet observed in radio and X-rays (see Morganti 2010 for a review), expanding in a set of radio lobes from $\sim 5 \mathrm{kpc}$ up to $\sim 250 \mathrm{kpc}$ (e.g. Israel 1998). At the center of Centaurus A there is an accreting supermassive black hole with a mass of $\sim 5 \times 10^{7} \mathrm{M}_{\odot}$, Israel et al. (2017) discovered an outflow of gas that from the center of Centaurus A extends along an axis close to that of the northern $\mathrm{X}$-ray/radio jet, detected in the neutral gas traced by CO and and C I, and in the far-infrared through fine-structure lines. Israel et al. (2017) detected also a less clear southern counterpart to the wellcollimated northern outflow. Furthermore, an [O III] ionization cone of $\sim 500 \mathrm{pc}$ along the minor axis, related to AGN or shock activities, is revealed by Bland-Hawthorn \& Kedziora-Chudczer (2003). This galaxy is the best example of a radio jet emitted by the central AGN interacting with the ISM, which may have induced star formation in the outskirts of the galaxy (positive feedback). The region where this interaction appears most clearly is characterised by filaments of highly ionized gas, between about 9 and $20 \mathrm{kpc}$ from the nucleus, roughly aligned along the jet direction (Morganti et al., 1991). Interestingly, these filaments are known to contain massive young stars, whose formation was possibly triggered by the interaction of the AGN radio plasma with a cloud of cold gas due to a recent merger, as suggested by the alignment with the jet direction and the kinematics of the gas (e.g. Santoro et al., 2016).

In this context, we investigated the central region of this galaxy, exploiting our approach of disentangling the disk from the outflow component to obtain an independent classification of their excitation properties with the BPT diagrams. The upper and lower panels of Fig. 4 shows the [N II] and [S II] BPT maps for the disk (on the left) and the outflow (on the right), with regions dominated by SF, AGN and composite/LI(N)ER activity indicated in blue, red, and green, respectively. The direction of the inner jet, revealed both in the radio and X-rays (see Fig. 5, Hardcastle et al. 2003) and consistent with the location of the bi-conical outflow dominated by LI(N)ER- and AGN-like ionization, is marked by the black arrow.

Looking back at Fig. 4, the SW portion of the galaxy FOV hosts a blob (solid circle, left pictures), characterised by a velocity consistent with the gas disk and by composite and SF dominated ionization ([N II]- and [S II]-BPT, respectively), that could be interpreted as SF triggered in the ionization cone due to compression of the galaxy ISM by the jet (as in Cresci et al. 2015a and Santoro et al. 2016). Moreover, a nearby clump appears to have SF like ionization, but velocities consistent with the gas in the outflow (solid square, right pictures), suggesting that in this case new stars could be formed at high velocity directly in the outflow (as in Maiolino et al. 2017). If confirmed, this would be the first example of these two types of positive feedback coexisting in the same object.

Fig. 6 shows the spectrum of the SF blob in the outflow (highlighted by the solid square in Fig. 4), that clearly presents outflow signatures. The asymmetric line profile can be reproduced by a three Gaussian component fit as shown in the figure. Fig. 7 illustrates the [N II] and [S II] BPT diagrams of these three components, indicated with the same color used in the previous figure. The redshifted 


\section{[NII] BPT map - Centaurus A}
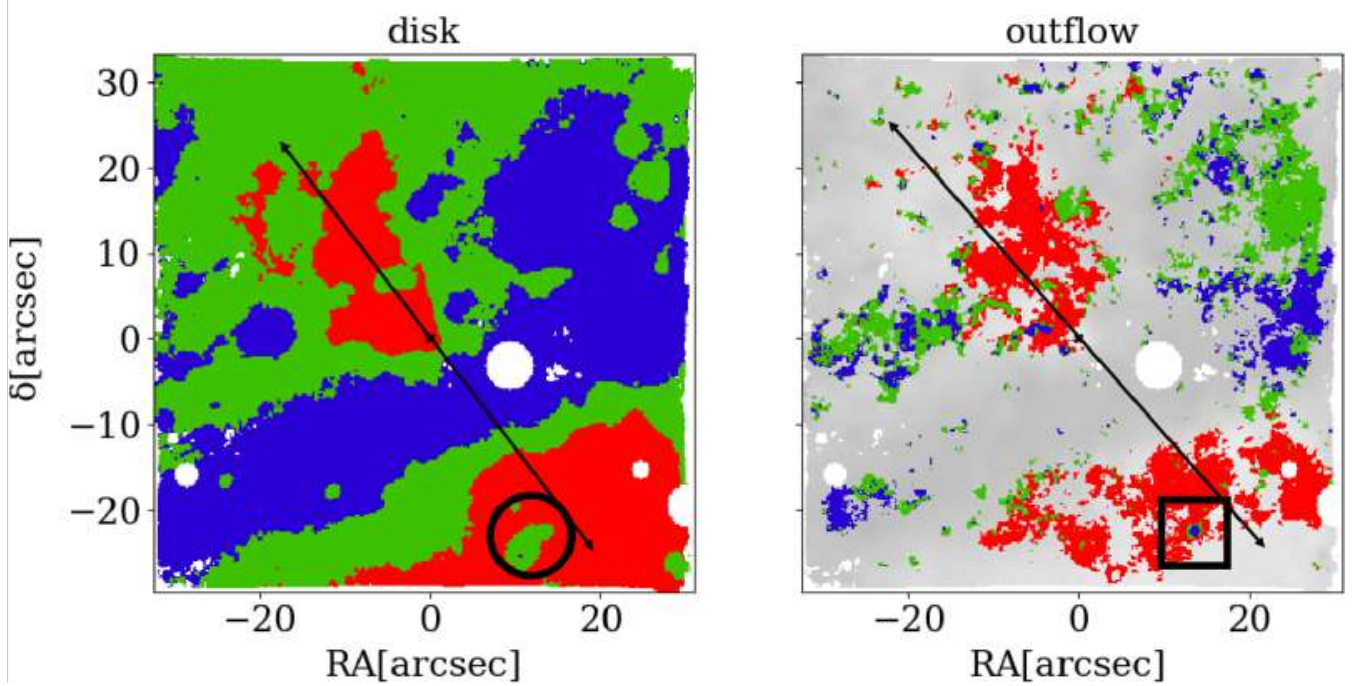

[SII] BPT map - Centaurus A
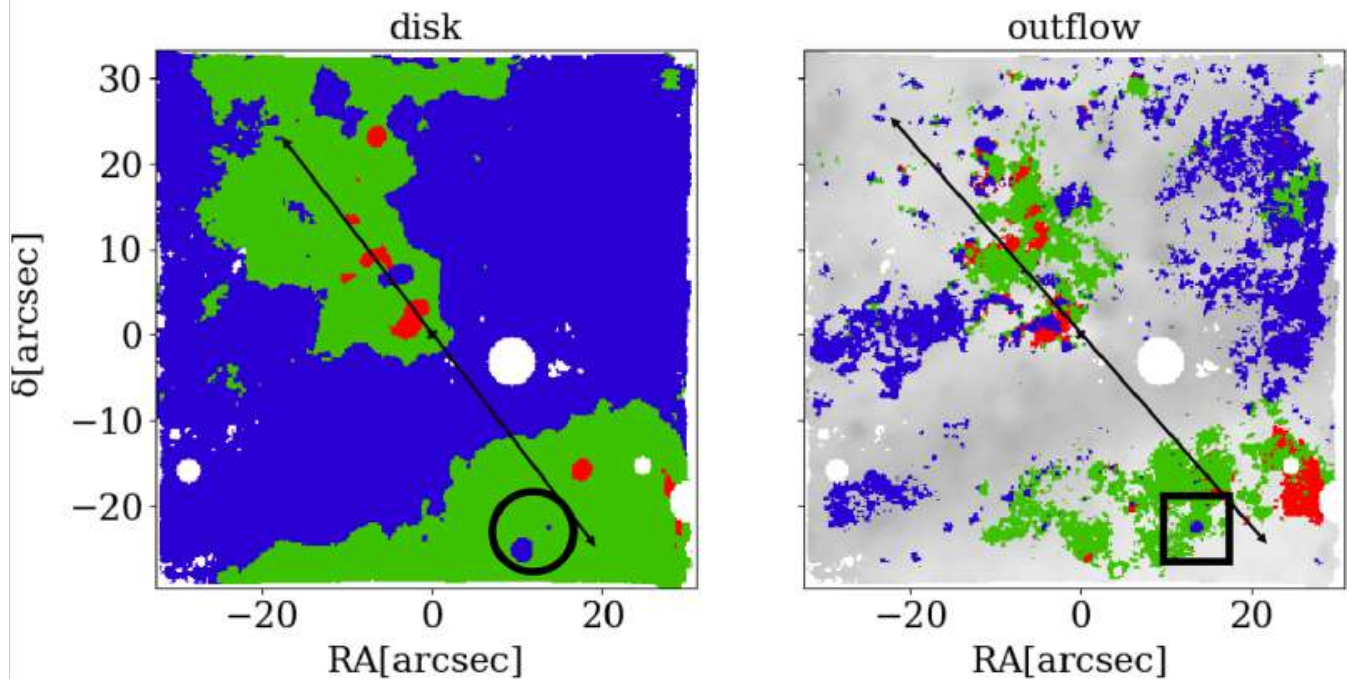

Figure 4. [N II]- (upper panel) and [S II]- (lower panel) BPT maps for the disk and outflow component of Centaurus A. To separate the two components, we divide each line profile in each spaxel of the MUSE FOV in velocity bins, associating to the disk the core of the line $(-150 \mathrm{~km} / \mathrm{s}<\mathrm{v}<+150 \mathrm{~km} / \mathrm{s})$ centred at Centaurus A systemic velocity $\left(\mathrm{v}_{\mathrm{sys}} \sim 547 \mathrm{~km} \mathrm{~s}^{-1}\right)$, and the sum of the redshifted and blueshifted wings to the outflow ( $\mathrm{v}>+200 \mathrm{~km} / \mathrm{s}-\mathrm{v}<-200 \mathrm{~km} / \mathrm{s})$. SF, AGN and Composite/LI(N)ER dominated regions are indicated in blue, red and green, respectively. The black arrow indicates the direction of the outflow, while the solid circle and square highlight the position of composite/SF blobs, located in the direction of the outflow.

Gaussian component (dashed red Gaussian) has a very strong $\mathrm{H} \alpha$ emission, with $\log ([\mathrm{N} \mathrm{II}] / \mathrm{H} \alpha) \sim$ $-0.6, \log ([\mathrm{S} \mathrm{II}] / \mathrm{H} \alpha) \sim-0.5$ and $\log ([\mathrm{O} \mathrm{III}] / \mathrm{H} \beta) \sim 0$ flux ratios, indicating SF ionization, pointing towards a stellar-like ionization source at redshifted velocities consistent with the outflow at the same location. On the other hand, the blueshifted (dashed-dotted blue Gaussian) and disk (dotted green Gaussian) components have AGN and composite/LI(N)ER-like ratios.

We estimate the star formation rate (SFR) of the two star forming blobs using the H $\alpha$-based calibration taken from Lee et al. (2009). From the extinction-corrected luminosity of the redshifted $\mathrm{H} \alpha$ component (shown in red in Fig. 6), we find a SFR $\sim 8 \times 10^{-3} \mathrm{M}_{\odot} / \mathrm{yr}$ in the outflow. Taking into account also the SF blob in the disk (highlighted in the black circle in Fig. 4, left panels), we obtain a total SFR $\sim 0.01 \mathrm{M}_{\odot} / \mathrm{yr}$, which is $\sim 3 \%$ of the global value of the galaxy $\left(\mathrm{SFR}=0.4 \mathrm{M}_{\odot} / \mathrm{yr}\right.$ DiamondStanic \& Rieke 2012). However, we are observing just a small part of the outflow in Centaurus A, 


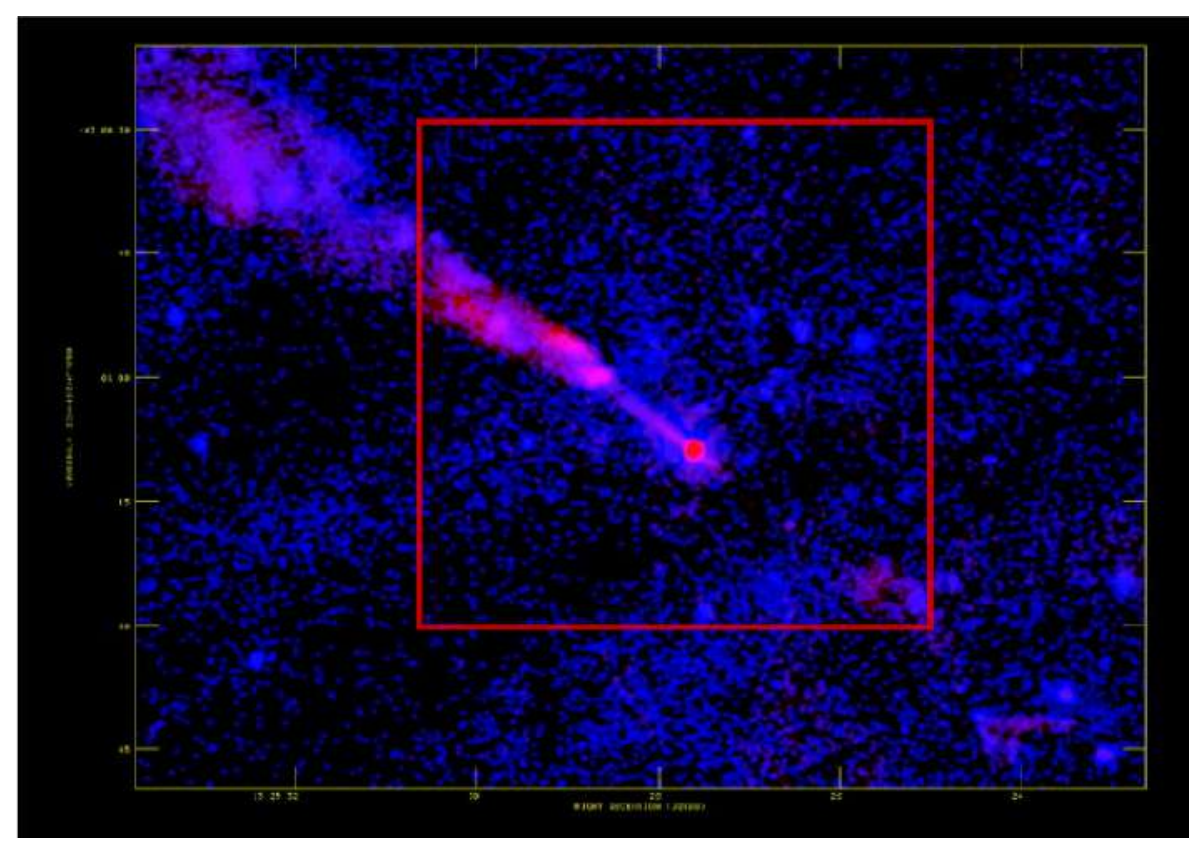

Figure 5. X-ray ( 0.4 and $2.5 \mathrm{keV}$, in blue) and radio structure (in red) of the jet in Centaurus A. This image is taken from Hardcastle et al. (2003). The red square superimposed indicates our MUSE FOV.

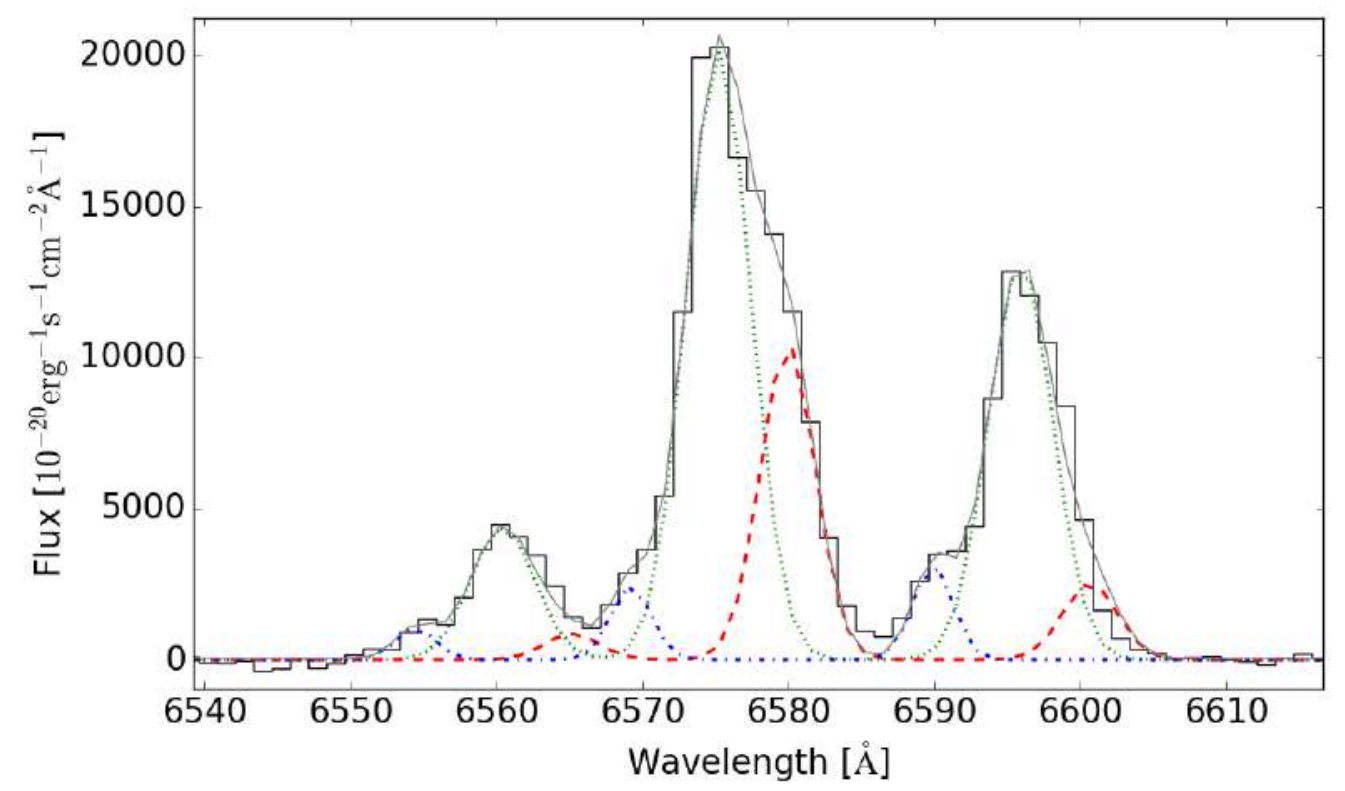

Figure 6. Spectrum of the SF blob in the outflow around $\mathrm{H} \alpha$ and $[\mathrm{N}$ II]. The asymmetric line profile is reproduced by a three Gaussian component fit: blueshifted (dashed-dotted blue Gaussian), systemic (dotted green Gaussian) and redshifted (dashed red Gaussian).

given the dust obscuration and the MUSE limited FOV due to the proximity of the source, which means that there could be many more SF clumps embedded in the outflow. Even if the contribution to the total SFR might seem irrelevant, it remains that Centaurus A may be used as a local test bench to explore in detail the structure and kinematic of the gas, improving our knowledge on positive feedback and possibly confirming that it is not a rare mechanism. Indeed, this phenomenon may play a significant role in the formation of the spheroidal component of galaxies at high redshift, where AGN-driven outflows are more prominent and, possibly, the associated SF inside those very massive outflows much higher (Gallagher et al., 2019).

Overall, these two blobs could represent the first evidence of the two modes of positive feedback 

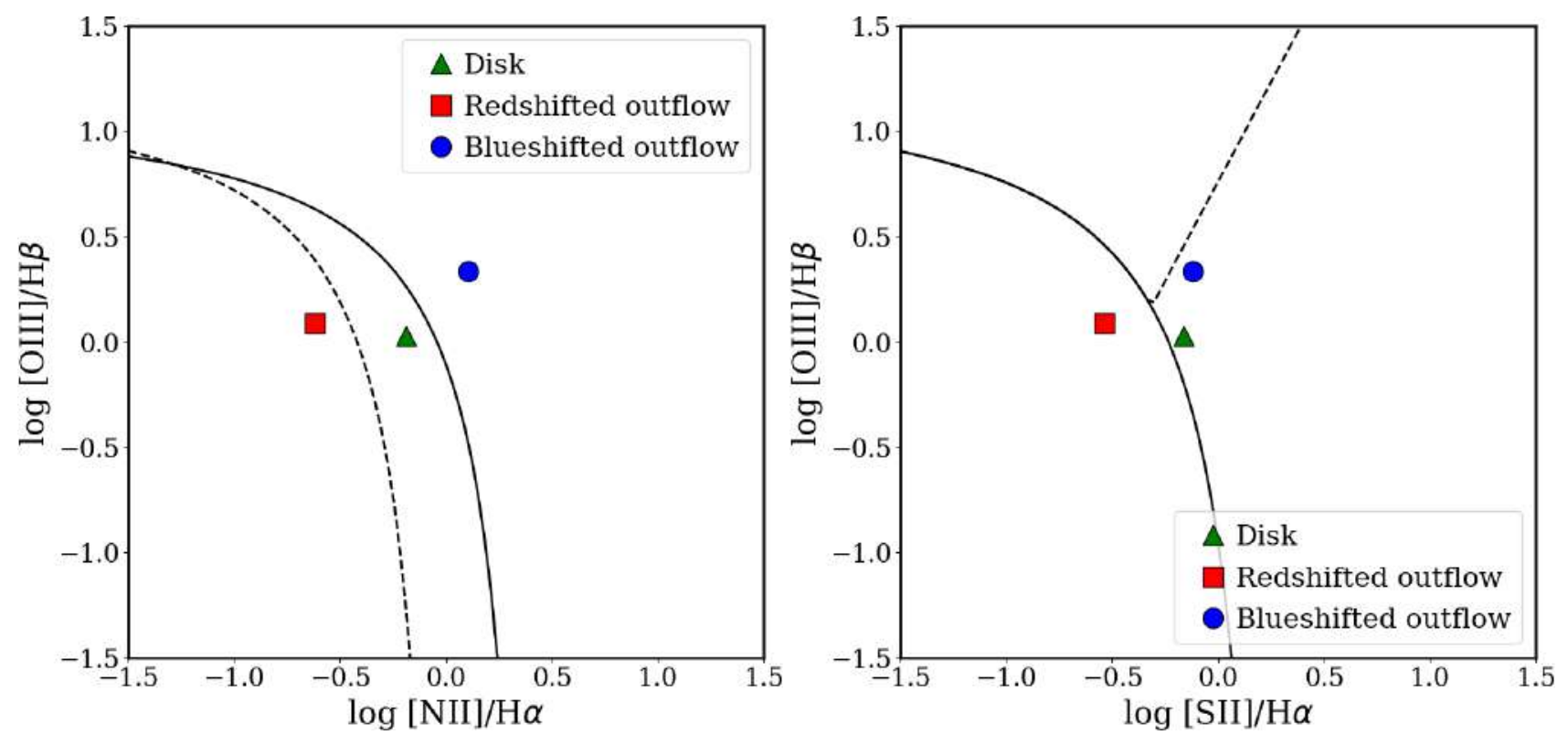

Figure 7. [N $\mathrm{NI}]$ and $[\mathrm{S} \mathrm{II}] \mathrm{BPT}$ diagrams of the three Gaussian components used to fit the asymmetric line profiles shown in Fig. 6. Note that while the blueshifted (dashed-dotted blue Gaussian) and disk (dotted green gaussian) components have AGN and Composite/LI(N)ER-like ratios, respectively, the redshifted component (dashed red Gaussian) is SF dominated.

in the nuclear region of this galaxy. However, Maiolino et al. (2017) demonstrated that only the combination of X-Shooter and MUSE data allow to undoubtedly identify the signatures of SF in the outflow. Indeed, $\mathrm{X}$-shooter has a broad-band spectral coverage able to cover IR diagnostics, such as the $[\mathrm{Fe}$ II $] \lambda 1.64 \mu \mathrm{m} / \mathrm{Br} \gamma \lambda 2.16 \mu \mathrm{m}$ versus the $\mathrm{H}_{2}(1-0) \mathrm{S}(1) \lambda 2.12 \mu \mathrm{m} / \mathrm{Br} \gamma$ and the $[\mathrm{Fe} \mathrm{II}] \lambda 1.25 \mu \mathrm{m} / \mathrm{Pa} \beta \lambda 1.28 \mu \mathrm{m}$ versus $[\mathrm{P}$ II] $\lambda 1.18 \mu \mathrm{m} / \mathrm{Pa} \beta$ diagrams (Oliva et al., 2001), that can further discriminate among SF, AGN and shock ionization. Typically, in star forming regions these ratios are expected to be very low, since shocks could enhance the emission of [Fe II] and $\mathrm{H}_{2}$, while the $\mathrm{Br} \gamma$ and the $[\mathrm{PII}]$ are direct tracers of the AGN UV and soft-X ionizing radiation, respectively. Moreover, X-shooter high spectral resolution allows to investigate the presence of young stars formed in the outflow. To do this, the stellar absorption features, such as the Balmer series down to $\lambda \sim 4000 \AA$ (tracing young hot O-B-type stars), the Ca II triplet at $\lambda \sim 8500 \AA$ (in case of recent $\mathrm{SF}$, dominated by young red supergiants and young asymptotic giant branch stars) and, possibly, the weak absorption feature of HeI $\lambda 4922 \AA$ (unambiguous tracer of B-type stars and of young stellar populations) can be taken into account. Hence, we successfully asked for observations with the X-Shooter spectrograph (0102.B-0292(A), P.I. Mingozzi). The observations were carried out during the nights of April 1-2 and of August 3-5 2019, making use of the 0.8 " slit, orientated in order to observe the two SF blob simultaneously, and will be analysed in the next months.

\section{Conclusions}

The MAGNUM survey is exploring the gas properties and the source of ionization of the outflowing gas in the NLR of the 9 nearby Seyfert galaxies, all characterised by prominent conical or biconical outflows. Exploiting the very high spatial resolution of the optical integral field MUSE spectrograph at VLT, we were able to disentangle the outflow component from the disk component in order to analyse its peculiarities through spatially and kinematically resolved maps. To do this, we divided the main emission lines $(\mathrm{H} \beta,[\mathrm{O} \mathrm{III}],[\mathrm{O} \mathrm{I}], \mathrm{H} \alpha$, [N II], [S II] and [S III]) in velocity bins, associating the core of the lines (centred on the stellar velocity in each spaxel) with the disc, and the blueshifted and redshifted wings with the outflow. In the following, we report our main results:

- We have analyzed the ionization state of the NLR of MAGNUM galaxies, by making spatially and kinematically resolved BPT diagrams. We find that the AGN/LI(N)ER-dominated 
outflow is characterised by the lowest and highest values of low-ionization line ratios (LILrs, $\log ([\mathrm{N} \mathrm{II}] / \mathrm{H} \alpha) \sim-1, \log ([\mathrm{S} \mathrm{II}] / \mathrm{H} \alpha) \sim-1$ and $\log ([\mathrm{N} \mathrm{II}] / \mathrm{H} \alpha) \sim 0.5, \log ([\mathrm{S} \mathrm{II}] / \mathrm{H} \alpha) \sim 0.5$, respectively), which are not observed in the disk. The lowest LILrs mainly come from the innermost regions of the outflowing cone, near the outflow axis. On the other hand, the highest LILr appear to come from the edges of the outflowing cones.

- We discussed the detection of two star-forming blobs embedded in the ionization cone of Centaurus A, that could represent the first evidence of the two modes of positive feedback in act in the nuclear region of this galaxy. We will soon analyse X-shooter data with the aim of unambiguously confirming the presence of positive feedback and fast moving young stars forming in the outflow.

We speculate that the gas in the outflowing cones of our galaxies is set up in clumpy clouds characterised by higher density and ionization parameters with respect to the disk gas. The innermost regions of the cone are optically thin to the radiation, being characterised by high excitation, and are possibly directly heated by the central ionizing AGN. The edges of the cones and the regions perpendicular to the outflow axis could instead be dominated by shock excitation probably because of the interaction between the outflowing gas and the ISM. Alternatively, these regions, generally characterised by low excitation $([\mathrm{S} \mathrm{III}] /[\mathrm{S} \mathrm{II}]<1)$ could be impinged by an ionizing radiation filtered by clumpy, ionized absorbers. The next step of our analysis will be a detailed modelling of each MAGNUM galaxy, also making use of the photoionization code CLOUDY (Ferland et al., 2017) to better investigate the different scenarios that we propose to interpret our findings. 


\section{References}

Belfiore F., et al., 2016, MNRAS, 461, 3111

Bland-Hawthorn J., Kedziora-Chudczer L., 2003, PASA, 20, 242

Cappellari M., Emsellem E., 2004, PASP, 116, 138

Cecil G., Dopita M. A., Groves B., Wilson A. S., Ferruit P., Pécontal E., Binette L., 2002, ApJ, 568, 627

Cresci G., et al., 2015a, AAP, 582, A63

Cresci G., et al., 2015b, ApJ, 799, 82

Diamond-Stanic A. M., Rieke G. H., 2012, ApJ, 746, 168

Dopita M. A., Sutherland R. S., 1995, ApJ, 455, 468

Ferland G. J., et al., 2017, RMXAA, 53, 385

Ferruit P., Wilson A. S., Mulchaey J., 2000, ApJS, 128, 139

Gallagher R., Maiolino R., Belfiore F., Drory N., Riffel R., Riffel R. A., 2019, MNRAS, 485, 3409

García-Burillo S., et al., 2014, AAP, 567, A125

Hardcastle M. J., Worrall D. M., Kraft R. P., Forman W. R., Jones C., Murray S. S., 2003, ApJ, 593, 169

Israel F. P., 1998, AAPR, 8, 237

Israel F. P., Güsten R., Meijerink R., Requena-Torres M. A., Stutzki J., 2017, AAP, 599, A53

Karouzos M., Woo J.-H., Bae H.-J., 2016, ApJ, 833, 171

Kauffmann G., et al., 2003, MNRAS, 346, 1055

Kewley L. J., Dopita M. A., Sutherland R. S., Heisler C. A., Trevena J., 2001, ApJ, 556, 121

Kewley L. J., Groves B., Kauffmann G., Heckman T., 2006, MNRAS, 372, 961

Klessen R. S., Glover S. C. O., 2016, Saas-Fee Advanced Course, 43, 85

Lee J. C., Gil de Paz A., Tremonti C., Kennicutt Robert C. J., Salim e. a., 2009”, ApJ, 706, 599

Lena D., et al., 2015, ApJ, 806, 84

Maiolino R., Mannucci F., 2019, AAPR, 27, 3

Maiolino R., et al., 2017, NAT, 544

Markwardt C. B., 2009, in Bohlender D. A., Durand D., Dowler P., eds, Astronomical Society of the Pacific Conference Series Vol. 411, Astronomical Data Analysis Software and Systems XVIII. p. 251

McElroy R., Croom S. M., Pracy M., Sharp R., Ho I.-T., Medling A. M., 2015, MNRAS, 446, 2186

McQuinn M., 2016, ARAA, 54, 313

Mingozzi M., et al., 2019, AAP, 622, A146

Morganti R., 2010, PASA, 27, 463

Morganti R., Robinson A., Fosbury R. A. E., di Serego Alighieri S., Tadhunter C. N., Malin D. F., 1991, MNRAS 
Oliva E., et al., 2001, AAP, 369, L5

Santoro F., Oonk J. B. R., Morganti R., Oosterloo T. A., Tadhunter C., 2016, AAP, 590, A37

Storey P. J., Zeippen C. J., 2000, MNRAS, 312, 813

Vazdekis A., Sánchez-Blázquez P., Falcón-Barroso J., Cenarro A. J., Beasley M. A., Cardiel N., Gorgas J., Peletier R. F., 2010, MNRAS, 404, 1639

Venturi G., Marconi A., Mingozzi M., Carniani S., Cresci G., Risaliti G., Mannucci F., 2017, Frontiers in Astronomy and Space Sciences, 4, 46

Venturi G., et al., 2018, A\&A, 619, A74

Westmoquette M. S., Clements D. L., Bendo G. J., Khan S. A., 2012, MNRAS, 424, 416 